Journal of Applied Analysis

Vol. 5, No. 2 (1999), pp. 187-196

\title{
UNIFORM PROPERTIES AND HYPERSPACES OF METRIZABLE SPACES
}

\author{
C. COSTANTINI and P. Vitolo \\ Received May 18, 1998 and, in revised form, October 9, 1998
}

\begin{abstract}
Two equivalent metrics can be compared, with respect to their uniform properties, in several different ways. We present some of them, and then use one of these conditions to characterize which metrics on a space induce the same lower Hausdorff topology on the hyperspace. Finally, we focus our attention to complete metrics.
\end{abstract}

\section{Introduction}

Throughout the paper, we will always denote by $X$ a metrizable topological space, by $\mathcal{M}(X)$ the set of all metrics which induce the topology of $X$, and by $\mathcal{C}(X)$ the collection of all closed subsets of $X$, including the empty set.

The symbol $\mathbb{N}$ will denote the set of natural numbers, not including 0 .

1991 Mathematics Subject Classification. 54E35, 54E15, 54B20.

Key words and phrases. Metrizable space, hyperspace, lower Hausdorff topology, Hausdorff metric topology, equivalent metrics, uniformly equivalent metrics, gap, totally bounded set, complete subspace, uniformly discrete set, wide family of sets.

ISSN 1425-6908 (c) Heldermann Verlag. 
Given $d \in \mathcal{M}(X)$ the $d$-distance of the point $x \in X$ from the set $A \in \mathcal{C}(X)$ is defined, as usual, by

$$
d(x, A)= \begin{cases}\inf _{a \in A} d(x, a) & \text { if } A \neq \emptyset ; \\ +\infty & \text { if } A=\emptyset .\end{cases}
$$

If $A, B \in \mathcal{C}(X)$ the $d$-excess of $A$ over $B$ is

$$
e_{d}(A, B)= \begin{cases}\sup _{a \in A} d(a, B) & \text { if } A \neq \emptyset ; \\ 0 & \text { if } A=\emptyset .\end{cases}
$$

If $A$ and $B$ are nonempty subsets of $X$ we define the $d-g a p$ between $A$ and $B$ as $D_{d}(A, B)=\inf _{a \in A} d(a, B)$; moreover we adopt the convention that $D_{d}(A, \emptyset)=D_{d}(\emptyset, A)=+\infty$ and $D_{d}(\emptyset, \emptyset)=0$.

The lower Hausdorff topology on $\mathcal{C}(X)$ relative to $d$, denoted by $\mathrm{H}_{d}^{-}$, is generated by all sets of the form

$$
\mathcal{S}_{d}^{-}(C, \varepsilon)=\left\{F \in \mathcal{C}(X) \mid e_{d}(C, F)<\varepsilon\right\}
$$

when $C$ runs through $\mathcal{C}(X)$ and $\varepsilon$ is a positive real number. The topology $\mathrm{H}_{d}^{-}$ is first-countable, and a sequence $\left(A_{n}\right)_{n \in \mathbb{N}}$ of closed subsets of $X$ converges to some $A \in \mathcal{C}(X)$ with respect to $\mathrm{H}_{d}^{-}$if and only if $\lim _{n \rightarrow \infty} e_{d}\left(A, A_{n}\right)=0$.

Similarly the upper Hausdorff topology on $\mathcal{C}(X)$ relative to $d$, denoted by $\mathrm{H}_{d}^{+}$, is generated by all sets of the form

$$
\mathcal{S}_{d}^{+}(C, \varepsilon)=\left\{F \in \mathcal{C}(X) \mid e_{d}(F, C)<\varepsilon\right\}
$$

when $C$ runs through $\mathcal{C}(X)$ and $\varepsilon$ is a positive real number. The Hausdorff (metric) topology is defined by $\mathrm{H}_{d}=\mathrm{H}_{d}^{+} \vee \mathrm{H}_{d}^{-}$.

It is known that two equivalent metrics $d$ and $\rho$ on $X$ give rise to the same Hausdorff and upper Hausdorff topology on $\mathcal{C}(X)$ if and only if they are uniformly equivalent (see for instance [1, Th. 3.3.2] or [2, Prop. 0.7]). Surprisingly enough, this is no longer true for lower Hausdorff topology (see Example 1.2 below); a weaker condition is needed, rather than uniform equivalence. The aim of this paper is to identify this condition.

In the sequel we shall use the following result.

Lemma 0.1 (Efremovič). Let $\left(x_{n}\right)_{n \in \mathbb{N}}$ and $\left(y_{n}\right)_{n \in \mathbb{N}}$ be two sequences on a metric space $(X, d)$; suppose that a positive number $\varepsilon$ exists such that $d\left(x_{n}, y_{n}\right) \geq \varepsilon$ for every $n \in \mathbb{N}$. Then there is a strictly increasing sequence $\left(n_{k}\right)_{k \in \mathbb{N}}$ of positive integers such that $d\left(x_{n_{h}}, y_{n_{k}}\right) \geq \varepsilon / 4$ for all $h, k \in \mathbb{N}$.

Proof. See [1, Lemma 3.3.1], or [5, Lemma 12.7] where a more general statement is proved. 


\section{Uniform properties of metrizable spaces}

Let $X$ be a metrizable space and $d, \rho \in \mathcal{M}(X)$; we consider the following properties:

(U0) The uniformity induced by $d$ is coarser than the one induced by $\rho$.

(U1) If $D_{d}(A, B)>0$, where $A, B \in \mathcal{C}(X)$ and at least one of them is not $d$-totally bounded, then $D_{\rho}(A, B)>0$.

(U2) If $D_{d}(A, B)>0$, where $A, B \in \mathcal{C}(X)$ and at least one of them is $d$-uniformly discrete, then $D_{\rho}(A, B)>0$.

(U3) Every $d$-uniformly discrete set is also $\rho$-uniformly discrete.

(U4) Every $\rho$-totally bounded set is also $d$-totally bounded.

Proposition 1.1. Each one of the statements listed above implies the subsequent one.

Proof.

$(\mathrm{U} 0) \Rightarrow(\mathrm{U} 1) \quad$ Let $D_{d}(A, B)=\varepsilon>0$. By assumption the identity map from $(X, \rho)$ to $(X, d)$ is uniformly continuous, thus there exists $\delta>0$ such that $d(x, y)<\varepsilon$ whenever $\rho(x, y)<\delta$. For every $a \in A$ and every $b \in B$, since $d(a, b) \geq \varepsilon$, we must have $\rho(a, b) \geq \delta$, and therefore $D_{\rho}(A, B) \geq \delta>0$.

$(\mathrm{U} 1) \Rightarrow(\mathrm{U} 2) \quad$ Let $A$ and $B$ be closed subsets of $X$ such that $D_{d}(A, B)>0$, where $A$ is $d$-uniformly discrete. If $A$ is finite then clearly $D_{\rho}(A, B)>0$; otherwise $A$ is not $d$-totally bounded and hence, by (U1), we have $D_{\rho}(A, B)>0$ again.

$(\mathrm{U} 2) \Rightarrow(\mathrm{U} 3) \quad$ Suppose there is a set $S$ which is not $\rho$-uniformly discrete but is $d$-uniformly discrete, with $d\left(s^{\prime}, s^{\prime \prime}\right) \geq \varepsilon>0$ for every distinct points $s^{\prime}, s^{\prime \prime} \in S$. First observe that $S$ remains not $\rho$-uniformly discrete when taking away any finite number of points (indeed, if $F$ is a finite subset of $S$ and $\inf \{\rho(x, y) \mid x, y \in S \backslash F, x \neq y\}=\delta>0$, then $\inf \{\rho(x, y) \mid x, y \in S, x \neq y\}=\min (\{\delta\} \cup\{\rho(x, y) \mid x, y \in F, x \neq$ $y\} \cup\{\rho(x, S \backslash F) \mid x \in F\})>0)$.

Now let $F_{0}=\emptyset$; inductively choose two distinct points $a_{n}, b_{n}$ in $S \backslash F_{n-1}$ such that $\rho\left(a_{n}, b_{n}\right)<1 / n$ and define $F_{n}=F_{n-1} \cup\left\{a_{n}, b_{n}\right\}$. The sets $A=\left\{a_{n} \mid n \in \mathbb{N}\right\}$ and $B=\left\{b_{n} \mid n \in \mathbb{N}\right\}$ are both $d$-uniformly discrete - hence closed - and $D_{d}(A, B) \geq \varepsilon$; but $D_{\rho}(A, B)=0$, contradicting (U2).

$(\mathrm{U} 3) \Rightarrow(\mathrm{U} 4)$ This follows from the obvious fact that a set $S$ is totally bounded if and only if every uniformly discrete subset of $S$ is finite. 
There are examples showing that the previously proved implications are not reversible.

Example 1.2. Let $X$ be the open interval $]-\pi, \pi[$ of the real line; for each $s, t \in X$ put $d(s, t)=|s-t|$ and $\rho(s, t)=\left|e^{i s}-e^{i t}\right|$ : then $d$ and $\rho$ are equivalent metrics which give rise to different totally bounded uniformities on $X$. Hence the implication (U1) $\Rightarrow(\mathrm{U} 0)$ does not hold.

Example 1.3. Let $X=A \cup E \cup \mathbb{N}$, where $A=\{-1 / n \mid n \in \mathbb{N}\}$ and $E=\{1 / n \mid n \in \mathbb{N}\}$. For every $x, y \in X$ define $\rho(x, y)=|x-y|$ and $d(x, y)=|f(x)-f(y)|$, where

$$
f(x)= \begin{cases}x, & \text { if } x>0 \\ x-1, & \text { if } x<0\end{cases}
$$

Both $d$ and $\rho$ induce the discrete topology on $X$.

Given any $d$-uniformly discrete set $S \subset X$ and any set $T \subset X$ disjoint from $S$, since $S \backslash \mathbb{N}$ must be finite, we necessarily have $D_{\rho}(S, T)>0$; on the other hand the set $B=E \cup \mathbb{N}$ is not $d$-totally bounded and $D_{d}(A, B)=1$, but $D_{\rho}(A, B)=0$. This shows that (U2) does not imply (U1), in general.

Example 1.4. Let $X=A \cup \mathbb{N}$, where $A=\{-1 / n \mid n \in \mathbb{N}\}$. For every $x, y \in X$ define $d(x, y)=|x-y|$ and $\rho(x, y)=|f(x)-f(y)|$, where $f(n)=n$ and $f(-1 / n)=n-1 / n$ for each $n \in \mathbb{N}$ : as in the previous example, both $d$ and $\rho$ induce the discrete topology on $X$.

Every $d$-uniformly discrete subset of $X$ contains at most finitely many elements of $A$, and therefore it is also $\rho$-uniformly discrete; on the other hand, $D_{d}(A, \mathbb{N})=\nVdash$, while $D_{\rho}(A, \mathbb{N})=\nvdash$. This shows that (U3) does not imply (U2), in general.

Example 1.5. Consider the set $X=\left\{s_{n} \mid n \in \mathbb{N}\right\}$ where, for each $n \in \mathbb{N}$, $s_{n}=\sum_{k=1}^{n} 1 / k$; for every $m, n \in \mathbb{N}$ let $d\left(s_{m}, s_{n}\right)=|m-n|$ and $\rho\left(s_{m}, s_{n}\right)=$ $\left|s_{m}-s_{n}\right|$. The totally bounded subsets of $X$ are the same for both metrics (namely the finite ones); on the other hand $X$ is uniformly discrete with respect to $d$, but not with respect to $\rho$. Hence (U4) does not imply (U3).

Proposition 1.6. Condition (U4) above is equivalent to the following: (U4') Every d-complete subspace is also $\rho$-complete.

\section{Proof.}


$(\mathrm{U} 4) \Rightarrow\left(\mathrm{U} 4^{\prime}\right) \quad$ Let $Y$ be a $d$-complete subspace; if $\left(y_{n}\right)_{n \in \mathbb{N}}$ is a $\rho$-Cauchy sequence, the set $\left\{y_{n} \mid n \in \mathbb{N}\right\}$ is $\rho$-totally bounded, hence $d$-totally bounded. Therefore a suitable subsequence $\left(y_{n_{k}}\right)_{k \in \mathbb{N}}$ is $d$-Cauchy, hence convergent; but then the whole sequence is convergent. It follows that $Y$ is $\rho$-complete.

$\left(\mathrm{U} 4^{\prime}\right) \Rightarrow(\mathrm{U} 4) \quad$ Consider any $\rho$-totally bounded set $Z$; if $Z$ is not $d$-totally bounded then it must contain a $d$-uniformly discrete sequence $\left(z_{n}\right)_{n \in \mathbb{N}}$. Since this sequence has no $d$-Cauchy subsequence, the set $\left\{z_{n} \mid n \in\right.$ $\mathbb{N}\}$ is a $d$-complete subspace, hence $\rho$-complete; but then it must be compact, and we get a contradiction.

\section{Hyperspaces of metrizable spaces}

In this section we give necessary and sufficent conditions under which the inequality $\mathrm{H}_{d}^{-} \leq \mathrm{H}_{\rho}^{-}$holds.

First, we need some preliminaries.

Lemma 2.1. Condition (U2) above is equivalent to the following:

(U2') Given two sequences $\left(x_{n}\right)_{n \in \mathbb{N}}$ and $\left(y_{n}\right)_{n \in \mathbb{N}}$ in $X$ - at least one of which is d-uniformly discrete - if $\lim _{n \rightarrow \infty} \rho\left(x_{n}, y_{n}\right)=0$ then $\lim _{n \rightarrow \infty} d\left(x_{n}, y_{n}\right)=0$.

Proof.

$(\mathrm{U} 2) \Rightarrow\left(\mathrm{U} 2^{\prime}\right) \quad$ Suppose that $\left(\mathrm{U} 2^{\prime}\right)$ is false: there are two sequences $\left(x_{n}\right)_{n \in \mathbb{N}}$ and $\left(y_{n}\right)_{n \in \mathbb{N}}$, one of which - say the former - is $d$-uniformly discrete, such that $\lim _{n \rightarrow \infty} \rho\left(x_{n}, y_{n}\right)=0$ while for some $\varepsilon>0$ we have $d\left(x_{n}, y_{n}\right) \geq \varepsilon$ frequently; we may assume, up to extract a subsequence, that $d\left(x_{n}, y_{n}\right) \geq \varepsilon$ for each $n \in \mathbb{N}$. Efremovič's lemma implies that, up to further extract a subsequence, we have $d\left(x_{m}, y_{n}\right) \geq \varepsilon / 4$ for every $m, n \in \mathbb{N}$. Now let $A=\left\{x_{n} \mid n \in \mathbb{N}\right\}$ and $B=\overline{\left\{y_{n} \mid n \in \mathbb{N}\right\}}$ : then $A$ is $d$-uniformly discrete, $D_{\rho}(A, B)=0$, and $D_{d}(A, B) \geq \varepsilon / 4>0$. Hence condition (U2) is not satisfied.

$\left(\mathrm{U}^{\prime}\right) \Rightarrow(\mathrm{U} 2) \quad$ If (U2) were false, there should exist two closed sets $A, B \subset$ $X$, where $A$ is $d$-uniformly discrete, such that $D_{d}(A, B)=\varepsilon>0$ but $D_{\rho}(A, B)=0$. Hence for each $n \in \mathbb{N}$ we may find $x_{n} \in A$ and $y_{n} \in B$ with $\rho\left(x_{n}, y_{n}\right)<1 / n$, which implies that $\lim _{n \rightarrow \infty} \rho\left(x_{n}, y_{n}\right)=0$, while $d\left(x_{n}, y_{n}\right) \geq \varepsilon$ for each $n \in \mathbb{N}$. It remains to prove that the set $\left\{x_{n} \mid n \in \mathbb{N}\right\}$ is infinite, so that the sequence $\left(x_{n}\right)_{n \in \mathbb{N}}$ has a $d$-uniformly discrete subsequence, which shows that (U2') does not hold. Suppose on the contrary that there is some $a \in A$ with $x_{n}=a$ for infinitely 
many $n$ 's: it follows that $\rho(a, B)=0$, i.e. $a \in B$; since $D_{d}(A, B)>0$, we have a contradiction.

Recall that a sequence $\left(A_{n}\right)_{n \in \mathbb{N}}$ converges to $A$ in $\mathcal{C}(X)$ with respect to $\mathrm{V}^{-}$, the lower Vietoris topology, if and only if for each open set $G \subset X$ such that $G \cap A \neq \emptyset$ we have $G \cap A_{n} \neq \emptyset$ eventually.

Lemma 2.2. Let $(X, d)$ be a metric space, and $A \in \mathcal{C}(X)$. Every sequence in $\mathcal{C}(X)$ which is $\mathrm{H}_{d}^{-}$-convergent to $A$ is $\mathrm{V}^{-}$-convergent, too, and if $A$ is $d$-totally bounded then the converse also holds.

Proof. The fact that $\mathrm{H}_{d}^{-}$-convergence implies $\mathrm{V}^{-}$-convergence is very well known (see for example [3]). For the remaining part of the statement, we refer to [2, Prop. 0.2] or [4, Lemma 4].

The following is our basic result: we are going to see that the inequality $\mathrm{H}_{d}^{-} \leq \mathrm{H}_{\rho}^{-}$is equivalent to one of the conditions listed in the previous section.

Theorem 2.3. Let $X$ be a metrizable space and $d, \rho \in \mathcal{M}(X)$. The topology $\mathrm{H}_{d}^{-}$on $\mathcal{C}(X)$ is coarser than $\mathrm{H}_{\rho}^{-}$if and only if condition (U2) holds.

Proof. In view of the previous lemma, we may prove that condition $\mathrm{H}_{d}^{-} \leq$ $\mathrm{H}_{\rho}^{-}$is equivalent to $\left(\mathrm{U} 2^{\prime}\right)$.

Suppose (U2') is false: so we can find a sequence $\left(x_{n}\right)_{n \in \mathbb{N}}$ which is uniformly discrete - i.e. there exists $r>0$ with $d\left(x_{m}, x_{n}\right) \geq r$ whenever $m$ and $n$ are distinct - and a sequence $\left(y_{n}\right)_{n \in \mathbb{N}}$ such that $\lim _{n \rightarrow \infty} \rho\left(x_{n}, y_{n}\right)=0$ while for some $\varepsilon>0$ we have $d\left(x_{n}, y_{n}\right) \geq \varepsilon$ frequently; we may assume, up to extract a subsequence, that $d\left(x_{n}, y_{n}\right) \geq \varepsilon$ for each $n \in \mathbb{N}$. Let $A=\left\{x_{n} \mid n \in \mathbb{N}\right\}$ and for each $k \in \mathbb{N}$, let $A_{k}=\left\{x_{n} \mid n \neq k\right\} \cup\left\{y_{k}\right\}$ : it is apparent that $e_{d}\left(A, A_{k}\right) \geq \min \{\varepsilon, r\}$ for every $k \in \mathbb{N}$, hence $\left(A_{k}\right)_{k \in \mathbb{N}}$ does not $\mathrm{H}_{d}^{-}$-converge to $A$; on the other hand $e_{\rho}\left(A, A_{k}\right)=\rho\left(x_{k}, A_{k}\right) \leq \rho\left(x_{k}, y_{k}\right)$ and therefore $\left(A_{k}\right)_{k \in \mathbb{N}}$ is $\mathrm{H}_{\rho}^{-}$-convergent to $A$, thus showing that $\mathrm{H}_{d}^{-} \not \leq \mathrm{H}_{\rho}^{-}$.

Conversely, suppose that $\mathrm{H}_{d}^{-} \not \leq \mathrm{H}_{\rho}^{-}$: then there exists $\left(A_{n}\right)_{n \in \mathbb{N}}$ in $\mathcal{C}(X)$ which converges to some $A \subset X$ with respect to $\mathrm{H}_{\rho}^{-}$, but not with respect to $\mathrm{H}_{d}^{-}$, so for a suitable $\varepsilon>0$ we have $e_{d}\left(A, A_{n}\right)>\varepsilon$ frequently and we may assume, up to extract a subsequence, that in fact $e_{d}\left(A, A_{n}\right)>\varepsilon$ for every $n \in \mathbb{N}$.

For each $n \in \mathbb{N}$, take $x_{n} \in A$ such that $d\left(x_{n}, A_{n}\right)>\varepsilon$. The set $T=\left\{x_{n} \mid\right.$ $n \in \mathbb{N}\}$ is not $d$-totally bounded, otherwise the sequence $\left(A_{n}\right)_{n \in \mathbb{N}}$, which $\mathrm{V}^{-}$-converges to $\bar{T}$, by Lemma 2.2 should also converge with respect to $\mathrm{H}_{d}^{-}$, 
and this is impossible. Thus, up to further extract a subsequence, we may assume that $\left(x_{n}\right)_{n \in \mathbb{N}}$ is $d$-uniformly discrete.

Since $\lim _{n \rightarrow \infty} \rho\left(x_{n}, A_{n}\right)=0$, for every $n \in \mathbb{N}$ we can find $y_{n} \in A_{n}$ in such a way that $\lim _{n \rightarrow \infty} \rho\left(x_{n}, y_{n}\right)=0$, too. On the other hand, $d\left(x_{n}, y_{n}\right) \geq$ $d\left(x_{n}, A_{n}\right)>\varepsilon$, hence condition (U2') is not satisfied.

For our second characterization of the inequality $\mathrm{H}_{d}^{-} \leq \mathrm{H}_{\rho}^{-}$we need to introduce some terminology.

Definition 2.4. Let $X$ be a metrizable space and $d \in \mathcal{M}(X)$. We say that a family $\mathcal{E}$ of subsets of $X$ is $d$-wide if

- the union of infinitely many members of $\mathcal{E}$ is not $d$-totally bounded;

$-\inf _{E \in \mathcal{E}} \operatorname{diam}_{d} E>0$.

Theorem 2.5. Let $X$ be a metrizable space and $d, \rho \in \mathcal{M}(X)$. The following are equivalent:

(i) $\mathrm{H}_{d}^{-} \leq \mathrm{H}_{\rho}^{-}$on $\mathcal{C}(X)$;

(ii) every $d$-wide family of d-totally bounded subsets of $X$ is also $\rho$-wide;

(iii) every $d$-wide family of compact subsets of $X$ is also $\rho$-wide;

(iv) every countable $d$-wide family of finite subsets of $X$ is also $\rho$-wide.

Proof. Assume (i) and suppose that $\mathcal{E}$ is a $d$-wide family of $d$-totally bounded subsets of $X$ which is not $\rho$-wide: in view of Theorem 2.3 and Proposition 1.1 (namely the implication (U2) $\Rightarrow(\mathrm{U} 4)$ ) we may assume that $\inf _{E \in \mathcal{E}} \operatorname{diam}_{\rho} E=0$. So, let $\left(E_{n}\right)_{n \in \mathbb{N}}$ be a sequence of pairwise distinct members of $\mathcal{E}$ with $\lim _{n \rightarrow \infty} \operatorname{diam}_{\rho} E_{n}=0$; as $E=\bigcup_{n=1}^{\infty} E_{n}$ is not $d$-totally bounded, there exists $\varepsilon>0$ and an infinite subset $H$ of $E$ such that $d(a, b) \geq \varepsilon$ whenever $a$ and $b$ are distinct elements of $H$. Since the members of $\mathcal{E}$ are $d$-totally bounded, none of them can contain infinitely many points of $H$ : therefore $H$ meets $E_{n}$ for infinitely many $n$ 's and up to extract a subsequence - we may suppose that $H \cap E_{n} \neq \emptyset$ for every $n \in \mathbb{N}$. Thus for each positive integer $n$ we may take $x_{n} \in H \cap E_{n}$ assuming, with no loss of generality, that $x_{m} \neq x_{n}$ whenever $m \neq n$, so that the sequence $\left(x_{n}\right)_{n \in \mathbb{N}}$ is $d$-uniformly discrete; if for every $n \in \mathbb{N}$ we take $y_{n} \in E_{n}$ such that $d\left(x_{n}, y_{n}\right) \geq(1 / 2) \operatorname{diam}_{d} E_{n}$, then $d\left(x_{n}, y_{n}\right) \geq \delta / 2$, where $\delta=\inf _{E \in \mathcal{E}} \operatorname{diam}_{d} E>0$. On the other hand $\lim _{n \rightarrow \infty} \rho\left(x_{n}, y_{n}\right) \leq$ $\lim _{n \rightarrow \infty} \operatorname{diam}_{\rho} E_{n}=0$, which shows that (U2') does not hold. By Theorem 2.3, we have a contradiction; hence (i) $\Rightarrow$ (ii).

In view of Theorem 2.3, to complete the proof it suffices to show that (iv) implies $\left(\mathrm{U} 2^{\prime}\right)$. So, suppose $\left(\mathrm{U}^{\prime}\right)$ is false: we have two sequences $\left(x_{n}\right)_{n \in \mathbb{N}}$ and $\left(y_{n}\right)_{n \in \mathbb{N}}$, one of which - say the former - is $d$-uniformly discrete, such 
that $\lim _{n \rightarrow \infty} \rho\left(x_{n}, y_{n}\right)=0$, but for a suitable $\delta>0$ we have $d\left(x_{n}, y_{n}\right) \geq \delta$ frequently, and there is no restriction in assuming that in fact $d\left(x_{n}, y_{n}\right) \geq \delta$ for each $n \in \mathbb{N}$. Letting $F_{n}=\left\{x_{n}, y_{n}\right\}$ for every $n \in \mathbb{N}$ defines a countable family $\mathcal{F}$ of finite sets. The union of any infinte subfamily of $\mathcal{F}$ cannot be $d$-totally bounded (because it contains infinitely many members of the sequence $\left.\left(x_{n}\right)_{n \in \mathbb{N}}\right)$ and $\inf _{n \in \mathbb{N}} \operatorname{diam}_{d} F_{n} \geq \delta>0$, so that $\mathcal{F}$ is $d$-wide. But $\mathcal{F}$ is not $\rho$-wide, as $\lim _{n \rightarrow \infty} \operatorname{diam}_{\rho} F_{n}=0$.

\section{Complete metrics}

In this section we will consider the case when $X$ is completely metrizable, and one of the metrics under consideration is complete.

Theorem 3.1. Let $X$ be a completely metrizable space and $d, \rho \in \mathcal{M}(X)$. If $d$ is complete then (U0) $\Leftrightarrow(\mathrm{U} 1) \Leftrightarrow(\mathrm{U} 2) \Leftrightarrow(\mathrm{U} 3)$.

Proof. We prove the implication (U3) $\Rightarrow(\mathrm{U} 0)$, and the result will follow from Proposition 1.1. Suppose (U0) is false; the identity map from $(X, \rho)$ to $(X, d)$ is not uniformly continuous, hence there exist $\varepsilon>0$ and two sequences $\left(x_{n}\right)_{n \in \mathbb{N}},\left(y_{n}\right)_{n \in \mathbb{N}}$ such that $\rho\left(x_{n}, y_{n}\right)<1 / n$ and $d\left(x_{n}, y_{n}\right) \geq \varepsilon$ for every $n \in \mathbb{N}$. By Efremovič's lemma we may assume, up to extract a subsequence, that

$$
\begin{array}{rl}
\forall n & \in \mathbb{N} \quad \rho\left(x_{n}, y_{n}\right)<\frac{1}{n} \\
\forall m, n \in \mathbb{N} & d\left(x_{m}, y_{n}\right) \geq \frac{\varepsilon}{4} .
\end{array}
$$

To complete the proof it suffices to show that there is a strictly increasing sequence $\left(n_{k}\right)_{k \in \mathbb{N}}$ of positive integers such that both the subsequences $\left(x_{n_{k}}\right)_{k \in \mathbb{N}}$ and $\left(y_{n_{k}}\right)_{k \in \mathbb{N}}$ are $d$-uniformly discrete, for in this case the set $\left\{x_{n_{k}} \mid k \in \mathbb{N}\right\} \cup\left\{\curvearrowright_{\ltimes_{\urcorner}} \mid \neg \in \mathbb{N}\right\}$ is $d$-uniformly discrete (because of (3.2)) but not $\rho$-uniformly discrete (because of (3.1)).

First, suppose that $\left(x_{n}\right)_{n \in \mathbb{N}}$ is $d$-totally bounded: up to extract a subsequence, we may assume that it is $d$-Cauchy; since $d$ is complete, $\left(x_{n}\right)_{n \in \mathbb{N}}$ converges to some $z \in X$ and, by (3.1), $\left(y_{n}\right)_{n \in \mathbb{N}}$ also converges to $z$, but this contradicts (3.2). Thus, there exists a subsequence $\left(x_{m_{h}}\right)_{h \in \mathbb{N}}$ of $\left(x_{n}\right)_{n \in \mathbb{N}}$ which is $d$-uniformly discrete. Then $\left(y_{m_{h}}\right)_{h \in \mathbb{N}}$ cannot be $d$-totally bounded (otherwise we get a contradiction by arguing in a similar way as we did above in case $\left(x_{n}\right)_{n \in \mathbb{N}}$ is $d$-totally bounded); again, there exists a subsequence $\left(y_{m_{h_{k}}}\right)_{k \in \mathbb{N}}$ of $\left(y_{m_{h}}\right)_{h \in \mathbb{N}}$ which is $d$-uniformly discrete. Putting $n_{k}=m_{h_{k}}$ for every $k \in \mathbb{N}$ gives the required strictly increasing sequence of integers. 
Corollary 3.2. Let $(X, d)$ be a complete metric space, and $\rho$ be a complete metric on $X$ which is equivalent to d. The topologies $\mathrm{H}_{d}^{-}$and $\mathrm{H}_{\rho}^{-}$coincide on $\mathcal{C}(X)$ if and only if $d$ and $\rho$ are uniformly equivalent.

Proof. It follows immediately from the previous result and Theorem 2.3.

Note that, even in case both $d$ and $\rho$ are complete, conditions (U3) and (U4) remain different, as shown in Example 1.5 above.

Remark 3.3. We have dealt with metric spaces only. But it is well-known that the Hausdorff topology may be defined on the hyperspace of a uniform space as well.

It turns out, as observed by one of the referees, that Theorem 2.3 admits a suitable reformulation for this more general situation. On the contrary, Corollary 3.2 does not extend to complete uniform spaces.

\section{References}

[1] Beer, G., Topologies on Closed and Closed Convex Sets, Kluwer, Dodrecht, 1993.

[2] Costantini, C. and Vitolo, P., On the infimum of the Hausdorff metric topologies, Proc. London Math. Soc. 70 (1995), 441-480,

[3] Francaviglia, S., Lechicki, A. and Levi, S., Quasi-uniformization of hyperspaces and convergence of nets of semicontinuous multifunctions, J. Math. Anal. Appl. 112(2) (1985), 347-370.

[4] Levi, S., Lucchetti, R. and Pelant, J., On the infimum of the Hausdorff and Vietoris topologies, Proc. Amer. Math. Soc. 118(3) (1993), 971-978.

[5] Naimpally, S. and Warrack, B., Proximity Spaces, Cambridge University Press, Cambridge, 1970 . 
Camilo Costantini

DipARTimento Di MATEMATICA

UNIVERSITÀ DI TORINO

via Carlo Alberto 10

10123 TORINO

ITALY
Paulo Vitolo

Dipartimento Di MATEMATiCA

UNIVERSITÀ DELLA BASILICATA

VIA NAZARIO SAURO 85

85100 Potenza

ITALY 